\title{
Influence of Carbon Nanotubes Added to a Commercial Adhesive
}

\author{
David Busek, and Pavel Mach \\ Department of Electrotechnology, Faculty of Electrical Engineering \\ Technická 2, 16627 Prague, Czech Republic, Phone: +420 224352355 \\ busekd1@fel.cvut.cz,mach@fel.cvut.cz
}

\begin{abstract}
This paper describes the influence of carbon nanotubes onto electrical properties of electrically conductive adhesives. This work is related to our previous research that described electrical connection network within electrically conductive adhesive and possible advantages of nonspherical particles distributed within the adhesive matrix. Two different commercial conductive adhesives were used in this experiment and different amounts of carbon nanotubes were mixed using different methods (ultrasound/rotation/spatula mixing) into the matrix. The positive effect in terms of mechanical properties can be expected according to previous research in the material field. The goal of this work is to describe the influence of this additional filler onto electrical parameters and generally to improve the adhesive properties.
\end{abstract}

\section{INTRODUCTION \& MOTIVATION}

Currently most used joining technique is soldering. However, soldering has its limitations when it comes to high pitch applications. Lead and lead free solders typically fail when scaled down to less than 100 micron pitch due to poor fatigue resistance. [2] Another option for joining electrical components is the use of electrically conductive adhesives (ECAs) that already have many advantages over solders. Among these are lower processing temperature, no need for fluxes \& possible fine pitch applications $(<0,1 \mathrm{~mm})$.

The disadvantages are, until now, lower electrical conductivity, lower thermal conductivity, worse long time stability, worse rework.

Despite these disadvantages, the electrically conductive adhesives are promising material for the future. In our previous research [1], theoretical study was done in order to set the way for the experiments. One of the ways to improve the properties of the conducting adhesive is to add conductive nanoparticles or nanotubes into the adhesive mix. For this experiment, multi walled carbon nanotubes were chosen.
A nanotube can be considered as a single sheet of graphite that has been rolled up into a tube. The electronic properties of the resulting nanotube depend on the direction in which the sheet was rolled up.

Some nanotubes are metals with high electrical conductivity (the electrical current that could be passed through a multi-wall nanotube corresponds to a current density of $10^{7} \mathrm{~A} / \mathrm{cm}^{2}$ [2]), while others are semiconductors with relatively large band gaps. The diameter of a multi-wall nanotube is tens of nanometers; for a single-wall nanotube it is one or two nanometers.

In 1998 Walt de Heer from the Georgia Institute of Technology in the US invented a way to measure the electrical conductance of multi-wall nanotubes. (see fig.2) A macroscopic fiber of multi-wall nanotubes is lowered into a drop of liquid metal and by dipping the nanotubes to different depths, the resistance of individual nanotubes and the resistance dependence on length can be determined.

The resistance dependence on length is very weak and therefore multi-wall nanotubes behave like ballistic conductors. [2] This is the most important finding for us and this property of the carbon nanotubes motivated us into our experiment. 


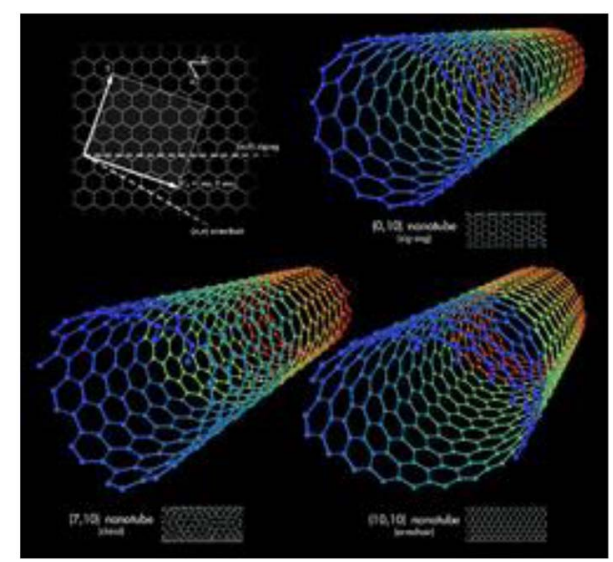

Fig. 1. Single wall nanotube [4]

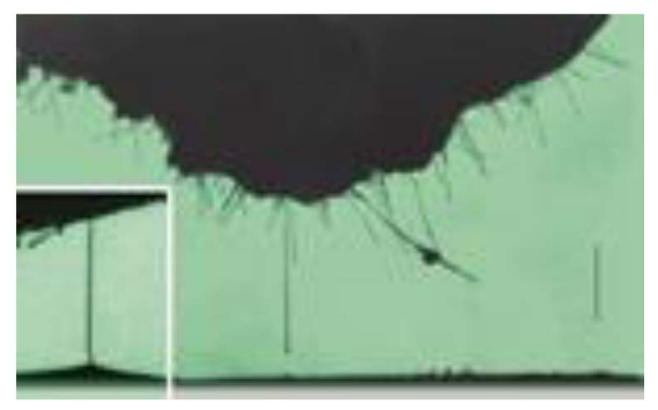

Fig. 2. Measuring the electrical conductance of multi-wall nanotubes [3]

\section{Measuring SeTUP}

In our experiments, two different adhesives from Amepox company in altogether seven modifications were used. The two basic conductive adhesives were chosen according to our previous research. The tested sample consists of seven serially interconnected resistors with zero nominal value.

Measured parameters were resistance and nonlinearity [3] of created joints. The cross-section of the joint is depicted in figure 3 .

As the joint should have the resistance as low as possible, the four-point probe method had to be used for the measurement.

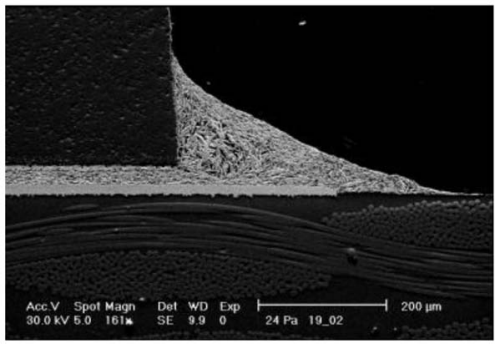

Fig. 3. Cross-Section of a tested conductive joint

The nonlinearity value is given by the spectral analyzer in $\mathrm{dBm}$ (decibel per miliwatt). As the goal of this work is to compare selected adhesive samples and not to measure the absolute value of the third harmonics, it is not necessary to recalculate $\mathrm{dBm}$ values to voltage. This would have been done using the following formula:

$$
\mathrm{U}=0,224 \cdot 10^{\left(\frac{\mathrm{U}_{\mathrm{dBm}}}{20}\right)}
$$

where $0 \mathrm{dBm}=1 \mathrm{~mW}$ on a $50 \mathrm{ohm}$ resistance, $\mathrm{I}=1 \mathrm{~A}$ and

$\mathrm{UdBm}=$ value given by the spectral analyser.

\section{ReSUlts}

Resistance measurement showed to be not very useful due to its lower sensitivity and therefore only nonlinearity results are presented in this paper.

Nonlinearity was evaluated for each connected resistor. As the inherent contribution of the component can be neglected (this was confirmed by measuring a soldered joint), the measured value represents nonlinearity of two joints. The graph on figure 4 (below) shows differences between different modifications of two basic adhesives. Due to $\mathrm{dBm}$ being measured in negative values, a higher column represents better modification (better electrical properties). A very good soldered contact exhibits a nonlinearity value of $-125 \mathrm{dBm}$ or higher, unmodified conductive adhesive has around $-100 \mathrm{dBm}$ and we consider anything around $\mathbf{- 8 0}$ or less as very bad. A comprehensive summary and description of the samples is shown in the table. 


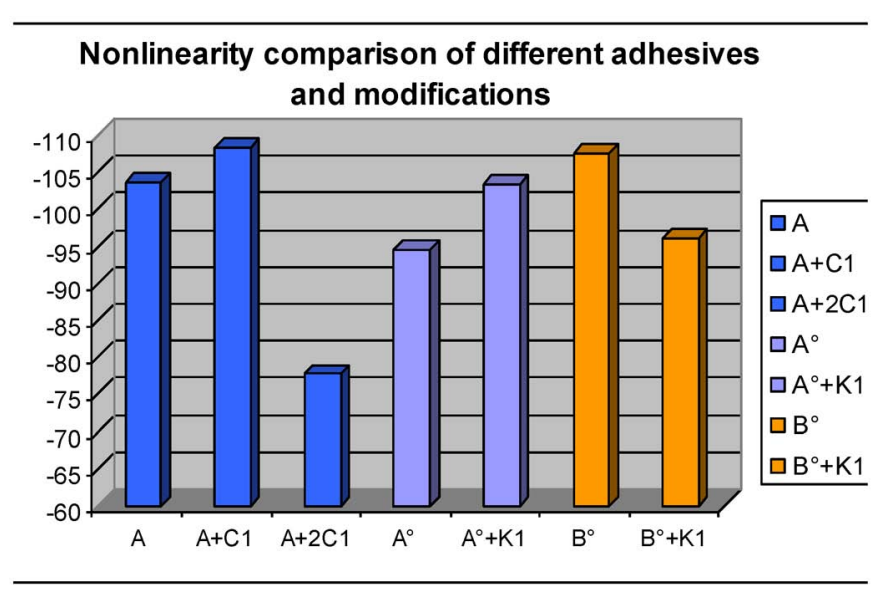

Fig. 4. Nonlinearity of different samples

A,B...basic adhesives

- ... spatula mixing

First 3 columns - ultrasound+rotary mixing

As the carbon is too light to be weighted, the "amount" was estimated experimentally. In future experiments, the amount will be determined using TEM picture of the cross-section. Amounts $\mathrm{C} 1$ and $\mathrm{K} 1$ are similar in percentage in the final adhesive, but as they were mixed into different amount of basic adhesive (and we were unable to weight it), it is an estimated value.
Unfortunately, only spatula mixing cross section is available at the moment, the dispersion of the carbon is not good and it is obvious that the carbon nanotubes create agglomerates - see figure 5 below. Carbon agglomerates are dark areas. A Detail of the dark area was also obtained and the nanotubes can be recognised (fig 6). It is expected, that the ultrasound dispersed the nanotubes better and that the nanotubes could create additional contacts between silver flakes in the adhesive, which should lead to better electrical properties of the mix. When double amount of nanotubes was added, the agglomerates were too big, the coherence was weakened and mechanical (and also electrical) properties were influenced down to an unusable level.

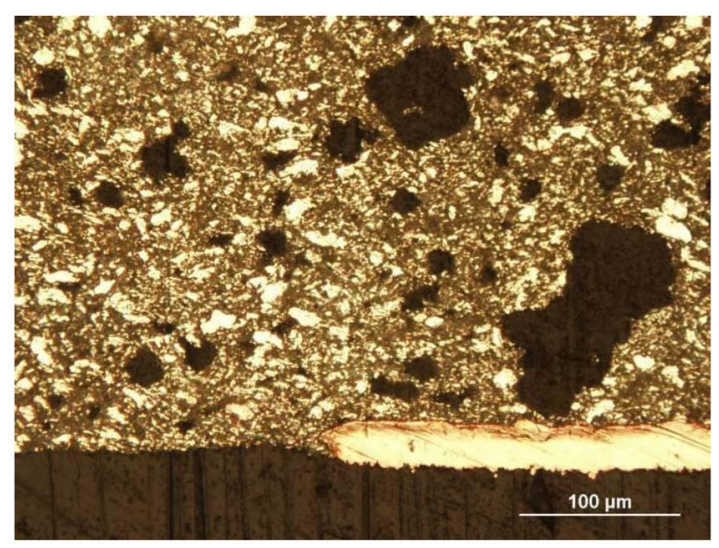

Fig. 5. Non-homogenous dispersion of the carbon.

\begin{tabular}{|c|c|c|c|}
\hline \multicolumn{4}{|c|}{ NONLINEARITY SUM- UP } \\
\hline Marking & $\begin{array}{c}\text { Real } \\
\text { composition }\end{array}$ & Mixing method & $\begin{array}{l}\text { Nonlinearity }(\mathrm{dBm})- \\
\text { the more negative } \\
\text { value, the better }\end{array}$ \\
\hline$A$ & $\mathrm{AX20}$ & ultrasound+rotary & $-103,6$ \\
\hline$A+C 1$ & $A \times 20+C 1$ & ultrasound+rotary & $-108,35$ \\
\hline$A+2 \times C 1$ & $A \times 20+2 \times C 1$ & ultrasound+rotary & $-77,7$ \\
\hline$A^{\circ}$ & $\mathrm{AX} 20$ & spatula only & $-94,6$ \\
\hline$A^{\circ}+K 1$ & $\mathrm{AX} 20+\mathrm{K} 1$ & spatula only & $-103,3$ \\
\hline $\mathrm{B}^{\circ}$ & AX70MN & spatula only & $-107,6$ \\
\hline $\mathrm{B}^{\circ}+\mathrm{K} 1$ & $\mathrm{AX70MN+K1}$ & spatula only & $-96,125$ \\
\hline
\end{tabular}

Tab. 1: Nonlinearity sum-up, $(\mathrm{C} 1=$ "amount1" of nanotubes, $\mathrm{K} 1$ = "amount2" -see explanation in text) 


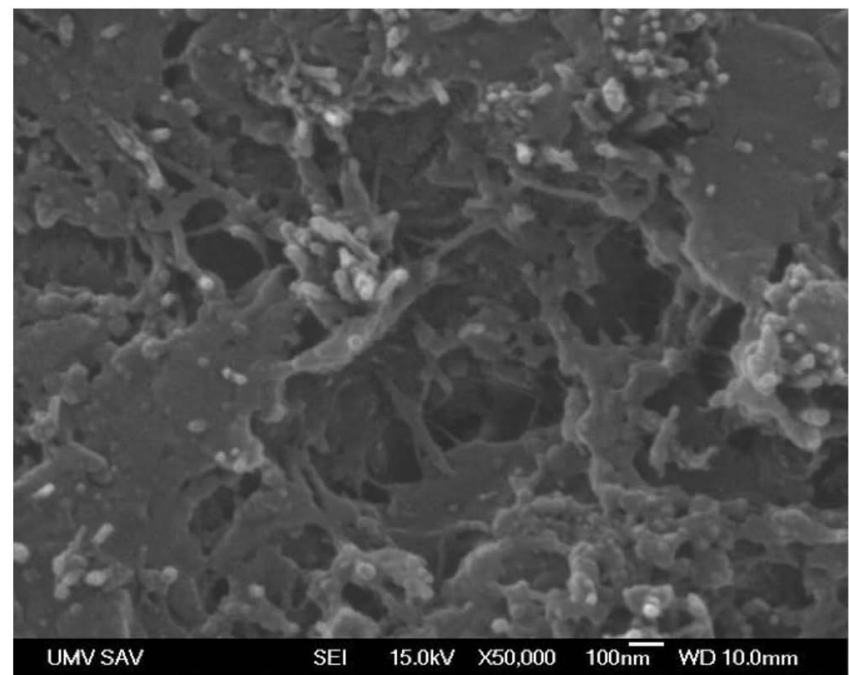

Fig. 6. Detail of the carnon nanotubes agglomerate.

\section{ConClusion}

According to the Table 1 and graph on Fig. 4, a small addition of carbon nanotubes improves not just mechanical toughness [5],[6], but can also positively influence the electrical parameters $\left(\mathrm{A} \rightarrow \mathrm{A}+\mathrm{C} 1\right.$; or $\left.\mathrm{A}^{\circ} \rightarrow \mathrm{A}^{\circ}+\mathrm{C} 1\right)$ as our research suggests. A too high (double) amount of nanotubes on the contrary significantly lowers electrical quality (third column: $\mathrm{A}+2 \mathrm{C} 1$ ). Not all adhesives react in the same way: AX70MN after addition of nanotubes lowers its quality $\left(\mathrm{B}^{\circ}+\mathrm{K} 1\right)$. An interesting conclusion from this experiment is also that ultrasound mixing does not create significantly different results from spatula mixing. Until now, it is not yet clear if the nanotubes create agglomerates after ultrasound mixing. The dispersion of the nanotubes in the matrix after spatula mixing is clearly visible from the cross-section of the adhesive (figure 5).

\section{REFERENCES}

[1] Bušek, David, Mach, Pavel, "Electrical connection network within an electrically conductive adhesive" In: ISSE 2008 - 31th International Spring Seminar on Electronics Technology [CD-ROM]. Budapest university of Technology and Economics, 2008, pp. 106-107. ISBN 978-963-06-4915-5.

[2] Matthias Heimann, Jorn Lemm, Klaus-Jurgen Wolter, "Experimental Investigations of Carbon Nanotubes / Epoxy Composites for Electronic Applications", XXXI International Conference of IMAPS, Poland, pp. 55$62,200$.

[3] Mach, P. - Papež, V. - Bušek, D. - Duraj, A. „Equipment for Measurement of Nonlinearity of Nominally Linear Components" In: 29th International Spring Seminar on Electronics Technology. Darmstadt: IEEE, 2006, vol. 1, pp. 240-243. ISBN 1-4244-0551-3.

[4] Wikipedia, the free encyclopedia: http://en.wikipedia.org/wiki/Carbon_nanotube (as of 10.2.2009)

[5] Sabyasachi Ganguli, Heshmat Aglan, Derrick Dean; „Microstructural Origin of Strength and Toughness of Epoxy Nanocomposites" in Journal of Elastomers and Plastics, Vol. 37, No. 1, 19-35 (2005)

[6] LIANG Shu-quan, JIA Chun-yan, TANG Yan, ZHANG Yong, ZHONG Jie, PAN An-qiang, „Mechanical and electrical properties of carbon nanotube reinforced epoxide resin composites" In Trans. Nonferrous Met. Soc. China 14, pp.675-679 (2007) 